\title{
Transatlantica
}

Revue d'études américaines. American Studies Journal

Révolution

\section{Little Miss Sunshine et le road movie}

\section{Anne Paupe}

\section{(2) OpenEdition}

Journals

Édition électronique

URL : http://journals.openedition.org/transatlantica/1187

DOI : $10.4000 /$ transatlantica. 1187

ISSN : 1765-2766

Éditeur

AFEA

Référence électronique

Anne Paupe, "Little Miss Sunshine et le road movie », Transatlantica [En ligne], 2 | 2006, mis en ligne le 19 janvier 2007, consulté le 29 avril 2021. URL : http://journals.openedition.org/transatlantica/1187 ; DOI : https://doi.org/10.4000/transatlantica.1187

Ce document a été généré automatiquement le 29 avril 2021.

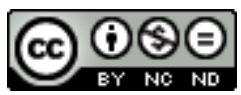

Transatlantica - Revue d'études américaines est mis à disposition selon les termes de la licence Creative Commons Attribution - Pas d'Utilisation Commerciale - Pas de Modification 4.0 International. 


\title{
Little Miss Sunshine et le road movie
}

\author{
Anne Paupe
}

1 Little Miss Sunshine est un road movie bien particulier : s'il relève assurément de ce genre, puisqu'il est essentiellement consacré au périple entrepris par ses protagonistes afin d'emmener, envers et contre tout, la petite Olive au concours de beauté qui l'attend en Californie, le film de Jonathan Dayton et Valerie Faris retient surtout l'attention par son humour satirique. Un rapide tour d'horizon de sa réception critique confirme qu'il a été rangé simultanément dans deux catégories génériques: le road movie et la comédie, cette dernière l'emportant largement. Ce film pose donc une double question : d'une part, celle du fonctionnement de ces deux registres, de l'autre, celle du statut du road movie, dont on se demande s'il est désormais voué à être subordonné à d'autres genres cinématographiques.

Le road movie, à ses débuts, n'était pourtant pas un genre comique : lorsqu'il a émergé avec Easy Rider (Dennis Hoppper, 1969), puis est devenu un genre autonome avec Cinq pièces faciles (Five Easy Pieces, Bob Rafelson, 1970), il pouvait certes donner lieu à des scènes comiques, mais là n'était pas son essence. La scène, dans le film de Bob Rafelson, où Jack Nicholson affronte la rigueur bornée d'une serveuse qui refuse d'adapter pour lui le menu, servait avant tout à souligner l'aliénation du personnage, tout en offrant un moment de comic relief. De même, si Point limite zéro (Vanishing Point, Richard Sarafian, 1971), Macadam à deux voies (Two-Lane Blacktop, Monte Hellman, 1971), L'Épouvantail (Scarecrow, Jerry Schatzberg, 1973) ou Sugarland Express (Steven Spielberg, 1974) pouvaient susciter le rire du public, ce n'étaient que des moments de répit. L'étude historique de la réception du road movie montre qu'il s'agit à l'origine d'un genre cinématographique considéré par les réalisateurs et par le public comme le moyen privilégié de représenter les travers de l'Amérique contemporaine. Le genre conserve cette visée idéologique lorsque Wim Wenders l'introduit en Europe avec Alice dans les villes (Alice in den Städten, 1974), Faux mouvement (Falsche Bewegung, 1975) et Au fil du temps (Im Lauf der Zeit, 1976). N'était Wenders, le road movie disparaît en tant que genre : durant plusieurs années, aucun film n'est perçu comme un road movie, mis à part 
quelques critiques qui évoquent ce genre à propos de Honkytonk Man (Clint Eastwood, 1982) et de Mad Max II (George Miller, 1982) ${ }^{1}$.

3 Ce n'est qu'avec la sortie parallèle en 1984 de Paris, Texas (Wim Wenders) et de Stranger than Paradise (Jim Jarmusch) que le genre suscite à nouveau l'intérêt des critiques. Le film de Jarmusch marque l'histoire du cinéma indépendant américain tout autant que celle du road movie: son humour pince-sans-rire et ses personnages décalés, bien plus que le sérieux mélodramatique du film de Wenders, conquièrent les critiques américains. Le road movie devient alors synonyme de comédie saugrenue, tendance qui se poursuit avec Leningrad Cowboys Go America (Aki Kaurismäki, 1989), jouant sur le contraste entre un groupe de rock finlandais d'inspiration folklorique et les chansons américaines découvertes en cours de route, ou avec Priscilla, folle du désert (Stephan Elliott, 1995) et $O$ Brother (O Brother, Where Art Thou, Joel Coen, 2000), mettant en scène les numéros extravagants joués par des personnages en complet décalage avec les régions rurales qu'ils traversent. Plus récemment, Broken Flowers (Jim Jarmusch, 2005) relève aussi de cette tendance : son protagoniste, présenté comme un ancien Don Juan, est en réalité un homme désabusé, qui n'a plus aucun désir et entreprend de rechercher son fils parce qu'il n'a rien de mieux à faire. Ce dernier exemple est emblématique de l'alliance d'humour grinçant et de tragique sous-jacent à beaucoup de road movies.

Les ingrédients habituels de cette veine comique du road movie se retrouvent dans Little Miss Sunshine: la galérie d'excentriques qui s'entasse dans le minibus Volkswagen en partance pour Redondo Beach rappelle les Leningrad Cowboys du film éponyme. Comme le groupe finlandais, la famille Hoover est traitée comme une unité dramatique, permettant une série d'oppositions et de cabales internes: l'alliance générale contre Richard, le père, et son obsession pour la " gagne ", est assortie de diverses oppositions familiales : entre le grand-père et l'oncle, puis entre le père et la mère, tandis que d'improbables complicités se forment entre le grand-père héroïnomane et sa petitefille qui se rêve en reine de beauté, ou entre l'oncle post-suicidaire et le neveu nihiliste. Le décalage entre les rêves de gloire entretenus par chacun des personnages (y compris, en un sens, Sheryl, la mère, qui rêve d'un monde de franchise et d'honnêteté) et leurs échecs accumulés renvoie aux revers essuyés avant eux par les héros de Priscilla, folle du désert ou de $O$ Brother.

5 Ce savant mélange d'éléments comiques a entraîné la notoriété de Little Miss Sunshine: son succès (hors compétition) auprès du public de Sundance a incité Fox Searlight à acquérir les droits de distribution du film pour la somme jusqu'alors sans précédent de dix millions de dollars (selon Variety, 21 janvier 2006). Le film a reçu le prix du public aux festivals de San Sebastian et de Sydney et, dernièrement, le Grand Prix du festival de Deauville. On y retrouve une des « recettes » du cinéma indépendant américain avec des personnages en marge des normes américaines de réussite financière, joués par des acteurs reconnus mais qui ne dominent pas la comédie hollywoodienne comme un Ben Stiller ou un Jim Carrey. Le film joue aussi sur quelques références culturelles européennes (Proust, Nietzsche) et sur des dialogues aux répliques acérées, à la limite $\mathrm{du}$ « politiquement correct» (dans une logique voisine des sitcoms les plus audacieuses : ainsi, lorsque l'oncle explique à Olive qu'il est tombé amoureux d'un de ses étudiants, celle-ci répond « That's silly » et le grand-père ajoute : « That's another word for it ».

6 Le road movie pourrait donc sembler n'être qu'un élément secondaire dans ce film. Toutefois, ses conventions génériques sont justement ce qui permet à la comédie d'éclore. Lors du huis-clos familial initial, les disputes éclatent à tout moment. L'oncle 
Frank est recueilli, et en fait surveillé par les Hoover, pour prévenir toute nouvelle tentative de suicide. Le fils Dwayne, admirateur de Nietzsche, a quant à lui fait vœu de silence, ce qui ne fait qu'accroitre les tensions. A l'exception de la petite Olive, soutenue par sa mère, la décision de partir pour Redondo Beach se fait à contre-cœur. Cependant, l'échappée sur la route permet un premier moment de comic relief décalé : à peine embarqués, le grand-père révèle son attirance pour les jeunes filles et confirme son penchant pour l'héroïne! La structure narrative du film est ensuite rythmée par les divers arrêts de la famille Hoover, au fil des incidents et accidents de parcours qui permettent de faire varier les rapports entre les personnages. Lors d'une première pause dans un fast-food, tous s'unissent contre les dérives autoritaires du père qui souhaiterait mettre sa fille au régime. À l'arrêt suivant, les tensions s'exacerbent entre les parents alors que l'oncle, occupé à sélectionner des revues pornographiques pour le grand-père, est abordé par son ex-petit ami. Pour clore le tout, la famille repart en oubliant Olive à la station-service. Olive est cependant récupérée au vol, et l'unité familiale préservée. Le minibus, dont la détérioration croissante reflète celle de la famille Hoover au bord du divorce, de la banqueroute et du suicide, comme le fait remarquer le fils, permet également la scène attendue de la panne, et par voie de conséquence les gags autour du démarrage. Ainsi, chaque fois que les personnages doivent pousser collectivement le minibus, l'oncle fait remarquer qu'il reste le plus grand spécialiste américain de Proust.

7 Finalement, la convention narrative du road movie selon laquelle les personnages entreprennent une quête de sens qui les transforme est respectée pour le fils (il sort de son mutisme), pour le père (il admet que son livre ne sera jamais publié) ou pour l'oncle (il reprend goût à la vie). La mort du grand-père, qu'on sait héroïnomane, ne surprend pas réellement. Rappelant le décès du grand-père Joad dans les Raisins de la colère, elle fait de Little Miss Sunshine une version moderne du film de John Ford, actualisée et transformée en comédie : la Californie, initialement rêvée comme un lieu paradisiaque par les personnages (ici, par Olive), devient le lieu de la désillusion. Enfin, selon la logique héritée du picaresque où le personnage traverse des milieux sociaux qui lui sont étrangers, Little Miss Sunshine extirpe la famille Hoover de leur milieu d'origine en les confrontant à la frénésie des autoroutes, puis à l'artifice inquiétant des concours de beauté pour fillettes, proposant une vision satirique de la société américaine.

Cependant, l'hybridité générique à l'œuvre dans Little Miss Sunshine donne la primauté à la comédie aux dépens du road movie. Plusieurs aspects essentiels du road movie sont absents du film. Ainsi, le film s'étend peu sur les grands espaces qui ont fait la fortune du genre. Lorsqu'il le fait, c'est pour accumuler les clichés, tel ce plan où le minibus, arrêté par un policier en raison d'un klaxon défectueux, est stationné devant des pompes à pétrole. Ce topos du road movie dénonce une réalité américaine, polluée et peuplée de personnages intolérants, qui n'est pas à la hauteur des mythes qui la représentent. Cette image renvoie également à d'illustres prédécesseurs : dans Cinq pièces faciles, le protagoniste est ouvrier pour l'industrie pétrolière et dans Thelma et Louise (Ridley Scott, 1991), le passage en Oklahoma montre des paysages envahis de pompes à pétrole. D'autre part, le film se concentre sur la cellule familiale, ne tirant guère parti de ces face-à-face atypiques avec des excentriques ou des psychopathes qui font le charme du road movie. Un des personnages rencontrés par hasard, l'ex-petit ami, a même une relation préexistante avec les protagonistes. La découverte de l'altérité se fait au sein de la famille, non par des rencontres extérieures. 
9 Little Miss Sunshine n'est pas sans rappeler Stranger than Paradise par sa concentration sur le huis-clos. Toutefois, le film de Jarmusch avait été perçu comme la résurgence du road movie, tandis que celui de Dayton et Faris a été décrit avant tout comme une comédie. Faut-il en conclure que c'en est fini du road movie? L'histoire de la réception du genre, hantée par des pronostics récurrents de sa mort et de sa renaissance, incite à la prudence. Ce film ne fait tout au plus que confirmer certaines caractéristiques du genre : sa tendance à l'hybridité, signe de la bonne santé du métissage dans le cinéma américain et l'affection qu'ont pour lui les cinéastes indépendants, même s'ils n'exploitent pas toutes ses ressources génériques. Enfin, l'engouement du public pour Little Miss Sunshine atteste qu'il a toujours soif des découvertes promises par tout road movie, attente dont ce film tire parti, recentrant l'aventure sur le cercle de la famille.

\section{NOTES}

1. Ceci selon le principe qu'un genre existe principalement grâce aux discours ambiants qui lui sont consacrés : si l'on n'en parle plus, c'est qu'il a disparu, même s'il est possible de retrouver les caractéristiques anciennement associées au genre dans certains films.

INDEX

Thèmes : Trans'Arts 